\title{
Computational Problems Concerning the Hilbert Matrix ${ }^{1}$
}

John Todd ${ }^{2}$

(November 21, 1960)

\begin{abstract}
The interaction between theoretical mathematics and practical computational expirement is illustrated by a discussion of recent work, at various centers, concerning the Hilbert matrix.
\end{abstract}

\section{Introduction}

Recent work, in various centers, concerning the Hilbert matrix shows very well the interaction between (theoretical) mathematics and practical computational experiments. Some aspects of this work will be discussed here in an expository manner. Although we shall be concerned mainly with the finite segments $H_{n}$ of the (infinite) Hilbert matrix

$$
H=\left\{h_{i, j}\right\}=\left\{(i+j+1)^{-1}\right\}, \quad i, j=0,1,2, \ldots,
$$

many of the results can be generalized, e.g., to matrices of the form

$$
H=\left\{\left(a_{i}+b_{j}\right)^{-1}\right\}, \quad i, j=0,1,2, \ldots, ;
$$

or

$$
H(\theta)=\left\{(i+j+\theta)^{-1}\right\}, \quad i, j=0,1,2, \ldots, \quad 0<\theta<1 .
$$

\section{Theory}

The literature of the last half-century contains many theoretical results concerning the Hilbert matrix. Of these only the basic inequality is immediately relevant [8, p. 234]:

2.1. If $\sum_{0}^{\infty} a_{m}^{2}$ is convergent, then

$$
\sum_{0}^{\infty} \sum_{0}^{\infty} a_{m} a_{n} /(m+n+1)<\pi \sum_{0}^{\infty} a_{m}^{2} .
$$

We note the following consequence of this.

2.2. The equation $H x=\pi x$ cannot be satisfied by any vector $x$ in Hilbert space, $l^{2}$.

Suppose

$$
H x=\pi x
$$

is satisfied for a vector $x \epsilon l^{2}$. Then it is permissible to multiply both sides by $x^{\prime}$, i.e., take inner products, to get

$$
x^{\prime} H x=\pi x^{\prime} x .
$$

1 This paper is based on an invited address to the Società Italiana per il Progresso delle Scienze, in Sicily in 1956; it was prepared with partial support from theOffice of Naval Research.

2 Present address: California Institute of Technology, Pasadena 4, Calif.
If $x=\left(x_{0}, x_{1}, \ldots . ..\right)$ so that $\Sigma x_{m}^{2}$ is convergent this gives

$$
\Sigma \Sigma x_{m} x_{n} /(m+n+1)=\pi \Sigma x_{m}^{2},
$$

in contradiction with (2.1).

The question as to whether there is an $x$, necessarily not in $l^{2}$, for which the equation

$$
H x=\pi x
$$

is true, was raised by O. Taussky [20]. This question will be discussed in sections 5 and 6 below.

\section{Applications}

Among the areas in applied mathematics in which the Hilbert matrix or related matrices have turned up are aerodynamics (A. R. Collar [3]), diffraction of electromagnetic waves (W. Magnus and F. Oberhettinger [12]), and statisties (I. R. Savage and E. Lukacs [17]).

\section{Computational Problems - Inversion}

Theoretically, the problem of inverting the finite matrices $H_{n}$ is solved because of the existence of an explicit representation of the inverse, a result which is, essentially, due to Cauchy. The matrix is of the form known as a double-alternant, and the inversion has been discussed e.g., by A. C. Aitken [1, p. 134], A. R. Collar [3], and G. Pòlya and G. Szegö [15, pp. 98-99, 299-300]. The explicit form of the inverse, and the actual numerical values for $n=1(1) 10$, have been given by I. R. Savage and E. Lukacs [17].

Practically, however, the problem remains interesting. For instance, although the Hilbert matrix itself turns up in idealized situations, it may be expected that slightly different matrices turn up in the practical circumstances which are approximated by these. It is, therefore, desirable to study the mechanical inversion of the Hilbert matrices $H_{n}$, especially as they enjoyed a reputation for illcondition i.e., "numerical instability," long before the advent of high-speed computers and the basic papers of von Neumann and Goldstine [25] and Turing [24], in order to compare the observed inverse with the theoretical one.

The Hilbert matrix and related ones (e.g., Lotkin [10]) have been often used to test matrix inversion 
programs prepared for high-speed computers. All the experiments of which we are aware [2, 16, 23] and in these several different schemes for inversion were used - completely confirm the bad reputation. The results quoted by Todd [23] are fully representative. For machines with 10 to 12 decimals, with floating point arithmetic to about 8 significant figures, there is rapid deterioration of the quality of the alleged inverses which are produced - and usually complete failure to produce any inverse for $H_{n}$ with $n$ about 8

A detailed examination of the condition of $H_{n}$ has been given by Todd [23]. Given a matrix $A$ and a specific process of inversion, the error in computing $A^{-1}$ can be found, in theory. In practice what is wanted is a cheap estimate of this, in terms of quantities associated with the matrix and e.g., the wordlength of the machine. Various condition-numbers have been introduced to measure the condition of the matrix. One of these, the $P$-condition number is defined as $P(A)=|\lambda| /|\mu|$ where $|\lambda|$ and $|\mu|$ are the greatest and least among the absolute values of the characteristic values, of $A$. It has been shown [23], that $\alpha$ being a certain positive constant,

$$
P\left(H_{n}\right)=O\left(e^{\alpha n}\right)
$$

while for the $n \times n$ matrix

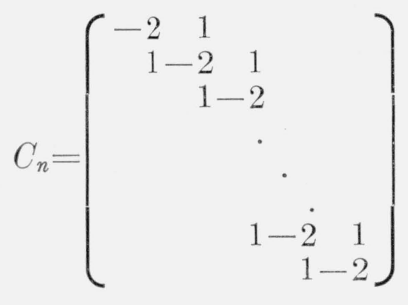

associated with a second-order differential equation, we have

$$
P\left(C_{n}\right)=O\left(n^{2}\right) .
$$

Using these results in the general error-estimate obtained by von Neumann and Goldstine [25], for an elimination process, and comparing these theoretical error-estimates with actual observed estimates, shows that the two are in reasonable agreement (see Todd [22]).

\section{Computation Problems - Characteristic Roots and Vectors}

As soon as programs for the computation of characteristic roots and vectors were constructed, it was natural to test them on the $H_{n}$, since input programs for this had already been constructed. There was no reason to suppose that it would be particularly difficult to handle- since it is considered that the presence of multiple roots, or roots close in absolute value would be the most likely source of trouble. The theory of positive matrices due to Perron and Frobenius (see e.g., Wielandt [26]) guar- antees the existence of a single dominant characteristic root with a characteristic vector all components of which are positive, so that the power method should be satisfactory. This is indeed the case and the dominant root, and the corresponding vector have been obtained for values of $n$ up to 200 at various centers $[5,18]$.

If more characteristic roots and vectors are needed, the Gantmakher and Krein [6] theory of completely positive matrices guarantees that the characteristic roots are all simple and positive and the components of the vectors exhibit a characteristic pattern: the first has all its components positive and each succeeding one has one more change of sign.

The last criteria shows up the weakness of the results obtained. For instance, a reasonably efficient code using floating point with 8 significant figures which gives the dominant root $\lambda_{5}$ of $H_{5}$ correct to $8 \mathrm{~S}$ gives the smallest root negative! Actually,

and

$$
\text { det } H_{5} \doteqdot 3.75 \times 10^{-12}
$$

$$
5.58 \times 10^{-6} \geq \mu_{5} \geq 1.12 \times 10^{-6}
$$

so the results are not too surprising.

The usual method for handling this case is an approximate diagonalization of the matrix using the Jacobi process, or the variation on this due to W. Givens [7] in which the matrix is reduced to triple diagonal form and the roots located by a Sturmian process. So far few actual results of the use of these methods have been published.

\section{More Theory}

Following experimental computations, O. Taussky [19] showed that, if $\lambda_{n}$ denotes the dominant characteristic root of $H_{n}$, then

$$
\lambda_{n}=\pi+O(1 / \log n)
$$

so that $\lambda_{n} \rightarrow \pi$. The actual approach to $\pi$ is very

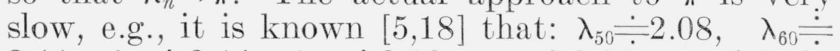
$2.11, \quad \lambda_{75} \doteqdot 2.14, \quad \lambda_{100} \doteqdot 2.18, \quad \lambda_{125} \doteqdot 2.21, \quad \lambda_{200} \doteqdot 2.27$; the exact order of magnitude of $\pi-\lambda_{n}$ does not appear to be known.

A constructive attack on the problem, already mentioned in section 2, of the existence of a vector $v$ satisfying

$$
H v=\pi \nu
$$

appeared difficult and an affirmative solution to it was obtained by T. Kato [9], who used an indirect approach. His method, which will now be described, was suggested by observations of the monotonic behavior of the coordinates of the dominant characteristic vectors of $H_{n}$, when these are normalized so that the first coordinate is unity.

Suppose the characteristic vector $v_{n}$ of $H_{n}$ corresponding to $\lambda_{n}$ is

$$
v_{n}=\left(v_{1}^{n}, v_{2}^{n}, \ldots, v_{n}^{n}\right), \quad v_{1}^{n}=1 .
$$


Numerical evidence suggests that both the sequence $\left\{\lambda_{n}\right\}$ and all the sequences $v_{i}^{i}, v_{i}^{i+1}, v_{i}^{i+2}, \ldots(i=1,2$, . . .) are monotone. The first fact is known (e.g., Collatz [4]) and the second is a special case of a more general result regarding the dominant vectors $a, b$ of two positive matrices $A, B$ where $A$ is $\alpha \times \alpha$ and $B$ is $\beta \times \beta$. If $B$ dominates $A$ in the sense that $\alpha \leq \beta$ and if $c_{i j}=b_{i j} / a_{i j}$ is a nondecreasing function of $i, j$ (as long as it is significant), then $b$ dominates $a$ in a similar sense, provided further that $B$ has all its second-order minors positive. This is proved using the fact (already noted) that in these circumstances the dominant vectors can be obtained by the "power" method. The special case used is that in which $A=H_{n}, \quad B=H_{n+1}$.

Now assume that $\lambda_{n}$ is bounded - that this is the case when we are dealing with $H_{n}$, follows from (2.1). Then $\lim \lambda_{n}=\lambda$, where $\lambda=\pi$ in the Hilbert case. It follows that the sequences $v_{i}^{i}, v_{i}^{i+1}, v_{i}^{i+2}, \ldots$ are bounded, for comparing the first coordinates in the equation

we have

$$
H_{n} v_{\eta}=\lambda_{n} v_{n}
$$

$$
h_{0, i} v_{i}^{n} \leq \sum h_{0, j} v_{j}^{n}=\lambda_{n} v_{1}^{n}=\lambda_{n} \leq \lambda,
$$

which gives, for all $n$,

$$
v_{i}^{n} \leq \lambda\left(h_{0, i}\right)^{-1} .
$$

In the Hilbert case this is

$$
v_{i}^{n} \leq \pi(i+1) .
$$

If we write $v_{i}=\lim _{n} v_{i}^{n}$, it can then be shown that the infinite vector $v=\left(v_{1}, v_{2}, \ldots\right)$ satisfies

$$
H v=\pi v .
$$

The result obtained by Kato [9], while answering the original question, itself suggests many more. For instance, is the vector $v$ the only (linearly independent) vector corresponding to $\lambda=\pi$ ? Are these characteristic values which exceed $\pi$ ?

\section{Recent Developments}

1. During the last few years the theory described in section 6 has been developed considerably by Tosio Kato and Marvin Rosenblum, and some of the questions raised there have been answered. Among the relevant results are the following:

M. Rosenblum [28] has shown that every complex number with a positive real part is a characteristic root of $H(\theta)$ for $\theta$ fixed, $\theta>0$. This was accomplished by using the theory of special functions to obtain a characteristic vector explicitly. In [29] Rosenblum completely determined the spectrum of $H(\theta)$, for any real $\theta \neq 0,-1,-2, \ldots$; this was accomplished using the Titchmarsh-Kodaira theory of singular differential operators.

T. Kato [30] shows that if $M(\theta)$ is the Hilbert bound of $H(\theta)$, i.e. $M(\theta)=\pi \operatorname{cosec} \pi \theta, \quad 0<\theta \leq \frac{1}{2}$;
$M(\theta)=\pi, \quad \theta \geq \frac{1}{2}$, then every $\lambda \geq M(\theta)$ is a characteristic value of $H(\theta)$, with a positive characteristic vector and there is no characteristic value $\lambda<M(\theta)$ which has a positive characteristic vector.

2. A reference to Hilbert's own work about $H_{n}$ is [27] where he evaluates det $H_{n}$. For some related results see R. B. Smith [32], W. W. Sawyer [33]. The problem of matrix inversion is discussed from the experimental point of view in [31], which includes further results on the Hilbert matrix.

\section{References}

[1] A. C. Aitken, Determinants and matrices (Edinburgh, 1951).

[2] S. E. Atta, Effect of propagated error on the inverse of Hilbert matrix. J. Assoc. Computing Machinery, 4, 36-40 (1957).

[3] A. R. Collar, On the reciprocal of a segment of a generalized Hilbert matrix. Proc. Cambridge Phil. Soc., 47, 11-17 (1951)

[4] L. Collatz, Einschliessungssatz für die charakteristischen Zahlen von Matrizen. Math. Z. 48, 221-226 (1942).

[5] G. E. Forsythe and M. Ascher, Unpublished computations on SWAC at Numerical Analysis Research, University of California, Los Angeles.

[6] F. Gantmakher and M. Krein, Sur les matrices complètement non-negatives et oscillatoires. Comp. Math. 4, 445-476 (1937).

[7] W. Givens, A method of computing eigenvalues and eigenvectors suggested by classical results on symmetric matrices, NBS Appl. Math. Series 29, pp. 117122 (1953).

[8] G. H. Hardy, J. E. Littlewood, and G. Pòlya, Inequalities (Cambridge, 1934).

[9] Tosio Kato, On the Hilbert matrix, Proc. American Math. Soc. 58, 78-81 (1957).

[10] M. Lotkin, A set of test matrices. Math. Tables Aids Comput. 8, 153-161 (1954).

[11] W. Magnus, Ueber einige beschränkte Matrizen. Archiv d. Math. 2, 405-412 (1949-1950).

[11a] W. Magnus, On the spectrum of Hilbert's matrix. Am. J. Math. $\mathbf{9 ,} 699-704$ (1950).

[12] W. Magnus and F. Oberhettinger, On systems of linear equations in the theory of guided waves. Comm. Pure and Appl. Math. 3, 393-410 (1950).

[13] J. C. P. Miller and R. A. Fairthorne, Hilbert's double series theorem and principal latent roots of the resulting matrix. Math. Tables Aids Comput. 3, 399-400 (1948).

[14] L. J. Paige and O. Taussky, Editors, Simultaneous linear equations and the determination of eigenvalues. NBS Appl. Math. Series 29 (1953).

[15] G. Pòlya and G. Szegö, Aufgaben und Lehrsätze aus der Analysis, II (Berlin, 1925).

[16] H. Rubenstein and J. D. Rutledge, High order matrix calculations on the UNIVAC. Proc. Assoc. Comput. Mach., pp. 181-186 (Pittsburgh Meeting, 1952)

[17] I. R. Savage and E. Lukacs, Tables of inverses of finite segments of the Hilbert matrix, NBS Appl. Math. Series 39, pp. 105-108 (1954).

[18] S. Schecter et al., Unpublished computations on UNIVAC at the Institute for Mathematical Sciences, New York Univ., New York, N.Y.

[19] O. Taussky, A remark concerning the characteristic roots of the finite segments of the Hilbert matrix, Quarterly J. Math. (Oxford) 20, 80-83 (1949).

[20] O. Taussky, Research problem 12, Bull. Am. Math. Soc. 60, 290 (1954).

[21] O. Taussky, editor, Contributions to the solution of systems of linear equations and the determination of eigenvalues, NBS Appl. Math. Series 39 (1954).

[22] J. Todd, The condition of certain matrices, II. Archiv d. Math. 5, 249-257 (1954). 
[23] J. Todd, The condition of the finite segments of the Hilbert matrix, NBS Appl. Math. Series 39, pp. 109 116 (1954).

[24] A. M. Turing, Rounding-off errors in matrix processes. Quart. J. Mech. Appl. Math. 1, 287-308 (1948)

[25] J. von Neumann and H. H. Goldstine, Numerical inverting of matrices of high order. Bull. Am. Math. Soc. 53, 1021-1099 (1947).

[26] H. Wielandt, Unzerlegbare, nicht negative Matrizen. Math. Z. 52, 642-648 (1950).

[27] D. Hilbert, Ein Beitrag zur Theorie des Legendreschen Polynoms, Acta Math. 18, 155-159 (1894); reprinted in Ges. Abh. 2, 367-370 (1933).

[28] M. Rosenblum, On the Hilbert matrix, I, Proc. Am. Math. Soc., 9, 137-140 (1958).
[29] M. Rosenblum, On the Hilbert matrix, II, Proc. Am. Math. Soc., 9, 581-585 (1958).

[30] T. Kato, On positive eigenvectors of positive infinite matrices, Comm. Pure Appl. Math., 11, 573-586 (1958).

[31] M. Newman and J. Todd, On the evaluation of matrix inversion programs, J. Soc. Indust. Appl. Math., 6, 466-476 (1958).

[32] R. B. Smith, Two theorems on inverses of finite segments of the generalized Hilbert matrix, Math. Tables Aids Comput. 13, 41-43 (1959).

[33] W. W. Sawyer, On the integral equation $k f(x)$ $=\int_{1}^{n}(x+y)^{-1} f(y) d y$, J. London Math. Soc. 34, 451453 (1959). 